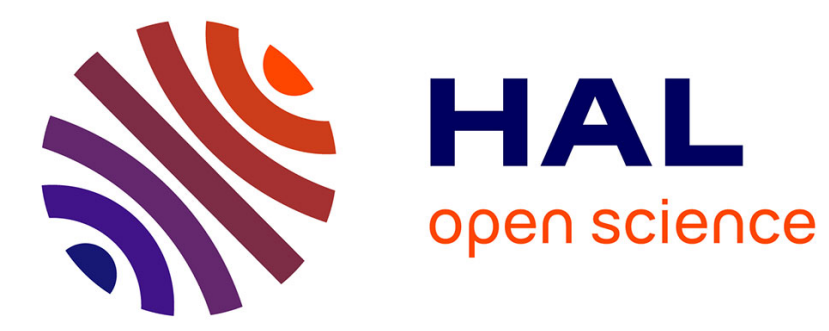

\title{
L'élargissement de 2004 au regard des précédents espagnol et portugais: un jeu de miroirs?
}

Bertrand Vayssière

\section{To cite this version:}

Bertrand Vayssière. L'élargissement de 2004 au regard des précédents espagnol et portugais : un jeu de miroirs?. Revue européenne d'histoire, 2009, 16 (4), pp.477-497. hal-00748552

\section{HAL Id: hal-00748552 \\ https://hal.science/hal-00748552}

Submitted on 5 Nov 2012

HAL is a multi-disciplinary open access archive for the deposit and dissemination of scientific research documents, whether they are published or not. The documents may come from teaching and research institutions in France or abroad, or from public or private research centers.
L'archive ouverte pluridisciplinaire HAL, est destinée au dépôt et à la diffusion de documents scientifiques de niveau recherche, publiés ou non, émanant des établissements d'enseignement et de recherche français ou étrangers, des laboratoires publics ou privés. 
«L'élargissement de 2004 au regard des précédents espagnol et portugais : un jeu de miroirs ? ».

RÉSUMÉ : Le but de cet article est de mettre en perspective les principales conséquences qu'ont eues l'intégration de l'Espagne et du Portugal à la CEE dans ces Etats, et de se demander quel élément, de l'Europe ou des pays eux-mêmes, a été le plus actif dans la dynamique de changement, politique aussi bien qu'économique. Il a également pour propos de s'appuyer sur ce précédent afin d'en tirer quelques leçons qui pourraient s'appliquer aux pays de l'Est, concernant leurs propres processus d'entrée dans l'Union européenne. Il s'agira ainsi de voir les différences et les ressemblances qui caractérisent ces deux élargissements. Enfin, l'article s'intéresse aux conséquences que l'entrée des pays d'Europe centrale et orientale de 2004 a entrainées pour les Etats ibériques eux-mêmes, du point de vue des politiques communautaires comme de celui de la place qu'occupent l'Espagne et le Portugal dans l’Union européenne élargie.

"The 2004 enlargement compared with the Spanish and Portuguese cases : a set of mirrors?". SUMMARY : The purpose of this article is to put into perspective the main consequences caused by the integration of Spain and Portugal into the EEC in these two States, and to ask what part, or of Europe or of the themselves, has been the most active in the dynamics of change, political as well as economic. It is also about relying on this precedent to draw some lessons that could be applied to Eastern Europe on their own process of entering the European Union. It will thus see the similarities and differences that characterize these two enlargements. Finally, the article focuses on the implications that the entry of countries of Central and Eastern Europe 2004 incurred for the Iberian States themselves, from the point of view of EU policies such as that of the place Spain and Portugal in the enlarged European Union.

Après avoir connu des décennies d'isolement relatif sous des régimes autoritaires, l'Espagne et le Portugal ont réussi la transition démocratique qui les a amenés avec succès au sein de la CEE. Cette expérience permet de jeter un regard objectif sur un passé récent et un processus original, qui concernent aussi bien les pays candidats que ceux qui les accueillent. Peut-on en tirer des leçons pour des cas plus contemporains, ainsi que pour d'autres qui ne tarderont pas à se présenter? Pour l'Espagne et le Portugal et leurs partenaires européens de l'époque, comme pour les pays de l'Est aujourd'hui, l'élargissement a eu de profondes conséquences et déclenché des processus complexes d'ajustement, dont l'historique et le bilan doivent être faits, dans le même temps où doit être dressé l'état des polémiques associées à l'idée d'élargissement, et qui ont effectivement eu lieu en 1986 : en la matière, les Européens, et singulièrement les Français, se sont toujours montrés « très en retrait quand il s'agit d'augmenter le nombre des convives autour de la table $»^{1}$. Certes, ces polémiques n'ont jamais atteint l'intensité provoquée par la candidature turque... Il n'y a ainsi pas eu de débat pour savoir si les pays ibériques appartenaient à l'Europe, et pas seulement pour des raisons géographiques : l'Espagne et le Portugal partagent, incontestablement, leur histoire, leurs traditions, leur culture, leur religion et leurs débats intellectuels avec le reste du Vieux continent, et les deux pays ont contribué à l'élaboration des valeurs européennes fondamentales. Leur entrée dans la Communauté européenne fut par conséquent une réaffirmation de ce fait. Dans le même temps, cependant, l'Espagne et le Portugal posaient de nombreux problèmes par la nature de leurs économies (en particulier l'agriculture), la sincérité de leur conversion à la démocratie et leur volonté de jouer une carte européenne qu'ils ne semblaient connaître que par les avantages qu'elle était susceptible d'apporter ${ }^{2}$, reproches depuis maintes fois formulés à l'égard des pays de l'Est. De plus, l'entrée de ces deux pays provoquait un certain bouleversement de la CEE en tant qu'acteur sur le Vieux continent, tout en réorientant une partie de sa mission : elle lui offrait une nouvelle dimension géopolitique, renforçant son Sud (et aussi ses poches de pauvreté), et

\footnotetext{
${ }^{1}$ Catherine Lalumière, ancienne secrétaire d'Etat aux affaires européennes, interviewée par Etienne Deschamps, Centre virtuel de la connaissance sur l'Europe, 17 mai 2006, http://www.ena.lu/mce.cfm

${ }^{2}$ Albert Broder, «Quelques réflexions sur l'entrée de l'Espagne dans la CEE avec une référence particulière à la France », Matériaux pour l'histoire de notre temps, année 1986, vol. 5, n5, pp.25-31.
} 
annonçait des liens plus étroits avec d'autres pays extra-européens, dont la place était jusqu'alors secondaire aux yeux de Bruxelles. En effet, l'élargissement ibérique a assuré un positionnement stratégique fort de l'Europe en Méditerranée et, à un moindre degré, en Amérique latine. Qui plus est, l'intégration ibérique a, pour des raisons économiques dues aux particularismes des deux pays entrants, mené à un développement plus rapide du système européen de cohésion et de solidarité, ce qui a pu déplaire à de nombreux observateurs. Un regard rétrospectif permet cependant d'affirmer que ces handicaps, en apparence insurmontables à l'époque où ils ont été mis en avant, ont été vaincus par les deux pays candidats : l'entrée dans la Communauté européenne a permis à l'Espagne et au Portugal d'influer sur des décisions européennes qui jusqu'ici leur échappaient, et c'est un fait que, depuis leur adhésion, tous deux ont joué un rôle important dans le processus de construction communautaire, redevenant des acteurs clés sur la scène européenne ${ }^{3}$.

La question est de savoir si ce processus, auquel nous nous sommes habitués au point de voir dans les cas ibériques des modèles que beaucoup de pays européens pourraient suivre, est réellement exemplaire. L'élargissement a-t-il vraiment induit un changement des systèmes politiques, économiques et sociaux de l'Espagne et du Portugal, au point que l'on pourrait parler d'un effet d'entraînement dû à l'appartenance à la Communauté européenne ? Ou bien cette conversion est-elle avant tout la conséquence d'un effort national, que l'Europe ne ferait qu'encourager et encadrer? Dans tous les cas de figure, peut-on tirer une leçon de ce précédent pour les pays d'Europe centrale et orientale qui ont constitué la dernière vague d'élargissement de l'Union européenne ? La comparaison n'est pas dénuée de sens, car le temps nous fait oublier combien les inquiétudes formulées à l'égard de l'intégration des PECO dans l'UE ont de ressemblances avec celles qui furent exprimées lors de l'entrée des pays ibériques dans la CEE. Il est intéressant, de plus, de connaître le positionnement de l'Espagne et du Portugal à l'égard de cet élargissement, qui signifie pour ces Etats un surcroît de concurrence.

Nous allons procéder en trois temps pour mener cette analyse : dans une première approche, nous ferons la synthèse des conséquences que l'intégration dans la Communauté européenne a entraînées pour les pays ibériques ; par la suite, nous examinerons les défis que l'élargissement de 2004 pose à l'Espagne et au Portugal ; enfin, nous tenterons d'analyser les quelques leçons que les pays de l'Est peuvent tirer de l'expérience lusohispanique.

\section{I- Espagne et Portugal face à l'Europe.}

a) Attitudes politiques et psychologiques.

La Communauté européenne a incontestablement joué un rôle important dans le parachèvement des processus de démocratisation au Portugal et en Espagne, même si le terme de «conditionnalité politique » n'est pas encore à l'ordre du jour ${ }^{4}$. Après tout, les élargissements précédents concernaient des pays correspondant aux standards politiques et économiques de la CEE historique (mis à part l'Irlande). C'est seulement dans la perspective des adhésions de la Grèce, de l'Espagne et du Portugal que le Conseil européen se sentît obligé d'adopter un texte politique relatif aux élargissements, contenant les exigences minimales relatives à la démocratisation des candidats. Celles-ci purent servir de levier au cours des premières phases du processus de négociations, utilisé en pleine conscience par différents hommes politiques. Les chefs d'Etat réunis à Copenhague le 8 avril 1978 déclarèrent ainsi «solennellement que le respect et le maintien de la démocratie représentative et des droits de l'homme constituent des éléments essentiels de l'appartenance aux Communautés »". Bruxelles avait donc défini explicitement les modalités institutionnelles que Madrid et Lisbonne devaient remplir, et les hommes politiques européens firent de même auprès de leurs homologues ibériques. Ainsi, la CEE a pu utiliser d'importants moyens de pression indirects au cours des négociations d'adhésion, qui ont duré la bagatelle de neuf années ${ }^{6}$,

\footnotetext{
${ }^{3}$ Pour une recension des principaux problèmes relatifs à l'entrée des deux pays dans la CEE, François Guichard et Bernard Lavallé, L'adhésion de l'Espagne et du Portugal à la CEE, Bordeaux, Maison des Pays ibériques, 1993 ; ouvr. coll., L'adhésion de l'Espagne et du Portugal à la CEE. Bilan et perspectives, Paris, CNRS éditions, 2000 ; Matthieu Trouvé, L'Espagne et l'Europe. De la dictature de Franco à l'Union européenne, Bruxelles, Peter Lang, à paraître.

${ }^{4}$ Edwige Tucny, L'élargissement de l'Union européenne aux PECO, Paris, L'Harmattan, 2000, pp.14-16. Voir la contribution maintenant classique de Aristide Zolberg, "L'influence des facteurs 'externes' sur l'ordre politique interne », in Madeleine Grawitz et Jean Leca (dir.), Traité de science politique, Paris, PUF, t.1, 1985, p.567-598.

«Au Conseil européen de Copenhague: déclaration sur la démocratie », Bulletin des Communautés européennes, $1978, \mathrm{n}^{\circ} 3, \mathrm{p} .5$.

${ }^{6}$ Les deux candidatures ont été déposées en 1977, respectivement le 28 mars pour le Portugal et le 28 juillet pour l'Espagne : « Demande d'adhésion du Portugal », Bulletin des Communautés européennes, mars 1977, n³, p.8 ;
} 
pour influer sur les décisions des hommes politiques et des acteurs économiques espagnols et portugais. La volonté de procéder par négociations, dont la longueur s'explique par la liste importante des exigences imposées par Bruxelles aux deux pays candidats, fut l'ultime levier aux mains de la CEE pour imposer le même cadre politique dans les deux pays ${ }^{7}$.

Parallèlement, l'entrée de l'Espagne et du Portugal eût un impact décisif sur les opinions de ces deux pays en favorisant «l'estime de soi nationale » et en liant cette image positive «à des sentiments démocratiques ${ }^{8}$. De plus, les contributions du budget européen (aides agricoles et aux régions), aussi bien que les bénéfices économiques de l'appartenance (les investissements étrangers) ont eu des effets matériels immédiatement perceptibles, permettant d'atténuer très rapidement certains des effets négatifs de la libéralisation et de la modernisation des structures économiques des deux pays. Des conditions de vie plus favorables et de meilleures perspectives de stabilité sociale et politique ont, à leur tour, influencé l'opinion publique et aidé à légitimer le nouveau système politique. L'adhésion à la CEE a forcé les pays ibériques à aligner leurs institutions sur le système communautaire, obligeant Madrid et Lisbonne à entreprendre des réformes administratives et la décentralisation, tout en tournant résolument le dos à toute autre tentation politique divergente : ainsi, le Portugal a réformé sa Constitution en 1989 afin de permettre la reprivatisation des compagnies qui avaient été nationalisées au cours de la révolution. Enfin, l'adhésion a également favorisé l'ouverture des élites sur l'extérieur et le développement de réseaux transnationaux, qui se sont révélés vitaux pour le renforcement des groupes d'intérêt et des partis politiques, tels les partis socialistes d'Espagne et du Portugal, qui reçurent un soutien substantiel de leurs homologues européens, ou les différents lobbyings régionaux espagnols ${ }^{9}$.

La victoire de la CEE serait-elle celle de la séduction, exercée par un modèle de démocratie portant haut l'épanouissement des libertés et le triomphe d'une certaine idée de l'économie de marché ? Quoi que l'on puisse dire, l'Europe, qui jusqu'ici n'avait pas été confrontée à l'idée d'intégrer des pays n'ayant pas connu la démocratie, jouait d'ores et déjà ce rôle de «pouvoir civil » dont parlait à l'époque François Duchêne ${ }^{10}$. Il serait cependant réducteur d'oublier, pour comprendre la réussite des transitions espagnole et portugaise, la part prise par les acteurs nationaux dans cette conversion démocratique. Les dynamiques internes furent décisives et le processus d'intégration européenne interagît avec toute une série de facteurs sociaux, politiques, et économiques antérieurs à l'adhésion : en Espagne, une dévolution radicale et sans précédent en faveur des régions autonomes a conduit à un Etat décentralisé, avec le développement des Autonomies ${ }^{11}$; au Portugal, après l'écroulement des tentatives révolutionnaires, l'Etat a également entrepris un processus systématique de modernisation sous la houlette de Mario Soares. Les deux transitions furent différentes, cependant. Pour Lisbonne, la route vers la démocratie commença par une réelle cassure (coup d'Etat du 25 avril 1974), pour Madrid, au contraire, la transition reposa sur un véritable consensus. Ces deux chemins vers la démocratie, parmi d'autres facteurs, ont entraîné des différences substantielles au sein des deux systèmes politiques ibériques en terme de développements institutionnels (ainsi, au Portugal, la décentralisation fut condamnée lors du référendum du 8

Ministerio de Asuntos Exteriores - Secretaría de Estado de Asuntos Europeos. Documentación : solicitud de adhesión. Madrid : [s.d.], pp.815-818.

${ }^{7}$ Geoffrey Pridham, «European Integration and Democratic Consolidation in Southern Europe », in Antonio Costa Pinto et Nuno Severiano Teixeira, Southern Europe and the Making of the European Union, New York, Columbia University Press, 2002, pp.188-189. Voir également l'attitude du Parlement européen, « Résolution du Parlement européen sur les négociations avec l'Espagne et le Portugal» (9 juillet 1982), Journal officiel des Communautés européennes, 13 décembre 1982, nC238, pp.94-95.

${ }^{8}$ Geoffrey Pridham, «The Politics of the European Community: transnational networks and democratic transitions in Southern Europe », in Geoffrey Pridham (ed.), Encouraging Democracy : the Institutional Context of Regime Transition in Southern Europe, Leicester, Leicester University Press, 1991, p.234.

${ }^{9}$ François Taulelle et Bertrand Vayssière, «Le sud-ouest européen : un espace transnational en formation ? », in Marie-Thérèse Bitsch (dir.), Le fait régional et la construction européenne, Bruxelles, Bruylant, 2003, pp.301322 ; Bertrand Vayssière, «La frontière franco-espagnole et l'Europe : le dépassement des limites ? », Annales du Midi, ${ }^{\circ} 259$, juillet-septembre 2007, pp.351-371.

${ }^{10}$ François Duchêne, «The European Community and the uncertainties of interdependence », Max Kohnstamm et Wolfgang Hager (eds), A Nation Writ Large? Foreign Policy Problems before the European Community, Mac Millan, Basingstoke, 1973 ; voir, dans la même veine, Johan Galtung, « Pax bruxellana : la superpuissance européenne », Prospectives, 1973, 1, pp.51-63. Le thème de la «puissance civile » a été récemment repris dans Zaki Laïdi, La norme sans la force. L'énigme de la puissance européenne, Paris, Presse de Sciences Po, 2005.

${ }^{11}$ William Genieys, «L'Espagne des Autonomies : les élites périphériques face à l'Etat », in Patrick Le Galès et Christian Lequesne (dir.), Les paradoxes des régions en Europe, Paris, La Découverte, 1997, pp.165-178 ; Berta Alvarez-Miranda, El Sur de Europa y la adhesión a la Comunidad : los debates políticos, Madrid, CIS, 1996 ; José Magone, «Attitudes of Southern European Citizens towards European Integration, in Antonio Costa Pinto et Nuno Severiano Teixeira (ed), op. cit., p.229. 
novembre 1998 par près de $63 \%$ de "non $»^{12}$ ), de performances économiques (l'Espagne a connu des taux de chômage plus importants tout au long de la période pré et post-adhésion ${ }^{13}$ ) et de projet collectif (le soutien des syndicats et des partis politiques à l'Europe est traditionnellement plus fort au Portugal qu'en Espagne $\left.{ }^{14}\right)$. De fait, l'intégration européenne n'a pas éliminé des différences majeures entre les deux pays ibériques, et le phénomène de l'intégration ne peut expliquer à lui seul les deux modèles de transformation politique propres à chacun d'eux. Ces différences résiduelles illustrent les limites des recherches qui ont voulu lier les transitions démocratiques des deux pays aux pressions extérieures et aux soutiens locaux à l'intégration européenne, et mettent en avant des cas particuliers qui échappent à toute tentative de modélisation ${ }^{15}$.

L'adhésion à la CEE a également été influencée par la culture politique de chacun des pays. Depuis la fin des régimes autoritaires, il y a toujours eu un soutien fort de l'opinion publique et des élites pour l'intégration des deux pays à la CEE comme moyen de consolider les nouveaux régimes démocratiques : démocratisation et intégration européenne paraissaient aux yeux des deux opinions faire partie du même processus, même si la première semblait être un préalable à la seconde. De plus, en encourageant l'investissement actif des deux pays au sein des institutions européennes, l'intégration européenne a permis de mettre fin à l'attitude « isolationnistefataliste » de la classe politique ${ }^{16}$. Les études d'opinion menées par Eurobaromètre et le CIS (Centro de Investigaciones Sociológicas) en Espagne ont montré le lien très fort qui unit le fonctionnement de la démocratie et l'idée d'Europe dans différentes enquêtes ${ }^{17}$; qui plus est, ce lien s'est constamment maintenu dans les deux pays depuis 1986, malgré quelques fluctuations. Le consensus presque unanime en faveur de l'intégration semble être la conséquence de la nécessité pour le Portugal et l'Espagne de dépasser l'isolement historique que les deux pays ont connu par rapport au reste de l'Europe depuis le XIXe siècle et jusqu'à la fin des régimes autoritaires dans les années $1970^{18}$.

Cependant, l'étude minutieuse des sondages offre un éclairage différent sur ce sentiment européen : celui-ci n'est pas inconditionnel, le consensus à l'égard de l'UE étant d'abord instrumental (surtout en Espagne). Le soutien à l'Europe semble coïncider avec les cycles économiques : bas durant les récessions économiques, et haut au dans les périodes de croissance. Certes, les niveaux de soutien à la démocratie comme régime politique légitime, préférable à toute autre alternative, sont restés élevés (autour de $80 \%$ des réponses dans les sondages), et Portugais et Espagnols se déclarent satisfaits du fonctionnement de leurs systèmes politiques ${ }^{19}$. Néanmoins, ces pays ont encore parmi les taux les plus faibles de participation aux diverses élections à l'ouest de l'Europe, et l'appartenance à des partis politiques et à des associations reste à un niveau peu élevé. En même temps, les citoyens n'ont pas le sentiment de pouvoir exercer une influence politique et expriment une opinion très ambivalente à l'égard des partis, qui se traduit par un faible intérêt pour la vie politique, ce dont l'Europe est l'une des principales victimes.

\section{2) Changements économiques et sociaux.}

L'intégration européenne fut un catalyseur pour la conversion finale des pays ibériques à l'économie de marché. Elle amena les acteurs politiques et économiques des deux pays à adopter les politiques et les stratégies allant de pair avec l'appartenance et l'acquis communautaires, qui consistait à adopter en même temps l'union douanière, la TVA, les politiques communes agricoles et de pêche, et les accords de commerce extérieurs déjà signés par la

\footnotetext{
${ }^{12}$ Yves Léonard, «Le référendum au Portugal : quel avenir ? », in Lusotopie 1999, Paris, Karthala, 1999, pp.4553.

${ }^{13}$ Gilles Saint-Paul, «L'anatomie du chômage en Espagne : une comparaison avec la France et les Etats-Unis », Economie et statistique, $\mathrm{n}^{\circ} 332-333,2000$, pp.159-167.

${ }^{14}$ Michèle Millot et Jean-Pol Roulleau, Les relations sociales en Europe, Paris, éd. Liaisons, 2005.

15 Robert M. Fishman, «Rethinking the State and Regime : Southern Europe's Transition to Democracy », World Politics, vol.42, Issue 3 (april), pp.422-440.

${ }^{16}$ José Magone, art. cit., p.225.

${ }^{17}$ Felix Moral, La opinión pública española ante Europa y los Europeos, Madrid, CIS, 1989. La dernière enquête Eurobaromètre sur « le futur de l'Europe » ( ${ }^{\circ} 251$, printemps 2006) démontre cependant un décrochage de plus en plus important entre l'Espagne (72\% d'opinions favorables à l'appartenance du pays à l'UE) et le Portugal (47\% seulement).

${ }^{18}$ Pour l'Espagne, voir les différentes contributions dans Richard Gillespie, Fernando Rodrigo, Jonathan Story (eds), Democratic Spain. Reshaping external relations in a Changing World, London and New York, Routledge, 1995; Matthieu Trouvé, “La diplomatie espagnole face à l'Europe (1962-1986). Enjeux, stratégies et acteurs de l'adhésion de l'Espagne aux Communautés européennes", in Michel Dumoulin, Antonio Ventura Díaz, Portugal y España en la Europa del siglo XX, Cuadernos de Yuste $n^{\circ} 3$, Yuste, Fundación Academia Europa de Yuste, 2005, pp.177-200.

${ }_{19}$ Voir, pour le cas espagnol, Eloisa del Pino et Jimena Larroque Aranguren, «Les Espagnols face à la démocratie. Continuité et changement dans la culture politique », Pôle Sud, 2005, n²3, pp.131-146.
} 
CEE. Certes, l'adhésion ne fut pas le seul moteur : la libéralisation économique, l'intégration commerciale et la modernisation des économies espagnole et portugaise avaient commencé dès les années 50 et 60 et les deux pays s'étaient rapprochés des normes européennes durant les deux décennies qui ont précédé l'adhésion à la CEE. En ce qui concerne l'Espagne, un plan de stabilisation fut appliqué dès 1959, pariant sur une ouverture partielle de l'économie nationale ainsi que sur une certaine libéralisation; après l'intégration du pays dans des instances internationales telles que l'OCDE, le FMI ou la Banque mondiale, un rapprochement fut opéré en direction de la CEE, culminant avec la signature, le 29 juin 1970, d'un accord commercial préférentiel abaissant les tarifs douaniers appliqués aux produits espagnols de $60 \%$ en moyenne contre $25 \%$ pour les produits européens en direction de l'Espagne. Cette asymétrie a pu profiter au pays, mais s'avéra vite insuffisante, à tel point que l'adhésion pure et simple s'imposa comme la seule vraie solution aux problèmes économiques nationaux. Cette dernière a en effet facilité les réformes micro et macro-économiques que les gouvernements ibériques successifs ont entreprises au cours des années 80 et 90, et qui étaient nécessaires à un basculement définitif vers l'économie de marché. Ainsi, l'adhésion a été un tremplin qui permit aux gouvernements ibériques de donner la priorité à la modernisation économique et de mener des politiques d'austérité (c'est-à-dire de réformer les marchés du travail et financier), dont le choc a pu être plus facilement absorbé du fait du consensus national. L'effort, loin de s'arrêter là, s'est poursuivi avec la décision prise par Lisbonne et Madrid d'adopter les critères de l'UEM définis à Maastricht, récompensés par la reconnaissance, de la part du Conseil européen de Bruxelles (3 mai 1998), que ces critères étaient bien respectés dans les deux pays ${ }^{20}$.

Ainsi, depuis 1986, l'intégration à la CEE conduit l'Espagne et le Portugal à l'adhésion au SME, à l'appartenance à l'UEM, et dans le même temps à l'introduction de la TVA et à la réduction des taxes sur les importations. Les réformes entraînées ainsi à la chaîne ont eut pour but d'assurer le bon fonctionnement des marchés de capitaux, l'efficacité des services publics, ainsi que la flexibilité sur le marché du travail: les marchés et les prix d'un certain nombre de biens et de services ont été dérégulés, un programme de privatisation a débuté dans les années 80 pour réduire la présence de l'Etat dans l'économie (c'est ainsi que le gouvernement espagnol a cédé des parts d'entreprises aussi prestigieuses que Telefónica, Endesa ou Repsol) et la concurrence a été adaptée aux exigences de la CEE. En termes statistiques, l'adhésion à l'UE a dynamisé le commerce dans le secteur manufacturier espagnol et portugais, mais a également entraîné plus de concurrence pour les entrepreneurs ibériques. Les difficultés de la transition ont été cependant limitées par le comportement des taux de change (avant l'UEM et la fin de certaines dévaluations compétitives) et par une augmentation très forte des investissements en provenance des pays de la CEE en Espagne et au Portugal, qui ont presque doublé entre 1985 et $1990^{21}$. Pour l'agriculture, la principale cause des problèmes d'ajustement a été le commerce, une plus grande pénétration des importations due à l'ouverture des marchés provoquant une contraction de la production nationale : ainsi, les peurs d'un effondrement commercial se matérialisèrent après l'adhésion (au profit d'autres membres de la CEE tels que la France et l'Italie), ce qui contribua à un exode rural croissant, dont les conséquences politiques ne furent pas négligeables ${ }^{22}$. L'un des défis les plus importants posés à l'adhésion de l'Espagne a ainsi été la diversification de son agriculture ${ }^{23}$.

L'intégration a cependant eu des effets dynamiques, qui ont renforcé certaines bonnes dispositions des économies ibériques. L'Espagne et le Portugal avaient à cet égard des atouts, tel qu'une base productive efficace, une force de travail peu chère et bien formée et l'accès à des marchés d'un grand potentiel. Parallèlement, l'entrée de ces deux pays dans la CEE réjouissait certains investisseurs non-Européens, tels que Japonais et Américains : l'une des conséquences de l'intégration a ainsi été l'énorme augmentation de l'investissement étranger en Espagne et au Portugal, passant de moins de $2 \%$ à plus de $6 \%$ du PIB de ces deux pays sur la dernière décennie. Un autre effet dynamique fut le renforcement de la compétitivité des entreprises ibériques en termes de compétences humaines, de stock en capital financier et technologique, tout cela même si des différences non négligeables demeurent avec les autres pays développés de la CEE.

L'Europe a incontestablement contribué à cette évolution. Entre 1994 et 1999, l'aide de l'UE a ainsi atteint 1,5\% du PIB espagnol et 3,3\% du PIB portugais; dans le budget 2004, les sommes allouées à l'Espagne (8,50 milliards d'euros) et au Portugal $(3,12)$ donnent un solde global par habitant largement positif $(300,4$ euros pour le Portugal et 200,1 pour l'Espagne ${ }^{24}$ ). Les fonds européens ont permis aux investissements publics de rester relativement stables à partir du milieu des années 80 : grâce à cela, l'infrastructure a été largement améliorée, et

\footnotetext{
${ }^{20}$ 98/317/CE : décision du Conseil du 3 mai 1998, Journal officiel de l'Union européenne, n ${ }^{\circ}$ L139 du 11 mai 1998, pp.30-35 ; Alfred Tovias, «The Southern European Economies and European Integration », in Antonio Costa Pinto et Nuno Severiano Teixeira (eds), op. cit., p.159-182.

${ }_{21}$ Bénédicte Larre et Raymond Torres, «La convergence est-elle spontanée ? Expérience comparée de l’Espagne, du Portugal et de la Grèce », Revue économique de l'OCDE, n 16, printemps 1991, p.214.

${ }^{22}$ François Guichard, «Le nouvel espace électoral portugais », Lusotopie, Karthala, 1996, pp.29-39.

23 R. C. Hine, «Customs Union Enlargement and Adjustment: Spain's Accession to the European Community », Journal of Common Market Studies, XXVIII/1, sept. 1989.

${ }^{24}$ Le Monde, Dossiers et documents, n³49, janvier 2006, p.1.
} 
les routes ainsi que les réseaux de télécommunication se sont développés aussi bien en nombre qu'en qualité. De plus, des dépenses ont été consacrées au système éducatif et à la formation, contribuant à une élévation du niveau de la force de travail.

L'enthousiasme doit cependant être tempéré, et il n'y a pas automaticité de la prospérité avec l'adhésion à l'Europe. Les vingt dernières années n'ont pas suffi pour rattraper le retard des économies espagnole et portugaise. L'intégration de l'Espagne et du Portugal a révélé des convergences et des divergences, nominales et réelles : depuis 1997, l'inflation en Espagne a dépassé la moyenne de l'UE chaque année (3,3\% en 2005 contre $2 \%$ pour la zone euro) ; au Portugal, la convergence réelle a ralenti chaque année depuis 1998, et est devenue négative à partir de 2000 : le pays a même été le premier Etat membre à être soumis à une procédure de déficit excessif. Le PIB par tête a connu une évolution cyclique dans les deux pays avec des augmentations significatives au cours des périodes d'expansion économique et de fortes baisses pendant les récessions, si bien que le «miracle » que l'on se plaît à décrire n'est pas permanent: depuis que l'Espagne a intégré la CEE en 1986, le revenu par tête a crû de $11,5 \%$ et de $14,2 \%$ au Portugal, alors qu'en Irlande, il a augmenté sans discontinuer dans le même temps de $38 \%$. Seule la Grèce, avec une augmentation de $6,8 \%$, avance à un rythme plus lent que l'Espagne et le Portugal. Ce différentiel de croissance explique les limites de la convergence, encore dans les années 90 où certains retards placent les deux pays dans une situation délicate au regard des performances moyennes dans l'UE : fort taux de chômage (15,4\% pour l'Espagne), faible taux de population active (autour de $50 \%$ dans les deux pays), formation insuffisante (seulement $28 \%$ des travailleurs espagnols ont un diplôme du supérieur, contre $56 \%$ en moyenne européenne), faible investissement dans le secteur de la Recherche et Développement et des technologies de l'information (le plus bas de l'UE), ainsi que des infrastructures qui restent inadéquates (le nombre de kilomètres pour 1000 habitants en Espagne représente seulement $47 \%$ de la moyenne européenne, $73 \%$ pour le réseau ferré). Tous ces chiffres ont depuis lors été améliorés, mais rappellent que le retard initial ne pouvait s'effacer de lui-même.

Du point de vue de la politique sociale, les années 80 représentèrent pour ces deux pays une décennie de stabilité politique qui, associée au développement des capacités financières et budgétaires de l'Etat, permirent une réelle augmentation des dépenses sociales (institutionnalisation du dialogue avec la signature de pactes sociaux). La dépense consacrée à la protection sociale était de 18,1\% du PIB en Espagne et de 12,8\% pour le Portugal en 1980, niveaux bien plus bas que la moyenne européenne d'alors, soit 24,3\%. Depuis l'adhésion, elle a significativement augmenté dans les deux pays. Cela dit, malgré une croissance de 3,4\% des ressources dédiées à la protection sociale en Espagne, le différentiel avec la moyenne européenne n'a pas été réduit : il est même passé de 6,2 points en 1980 à 6,8 en 1997. Le Portugal, parti avec des niveaux de protection sociale plus faibles, a eu plus de succès en réduisant le différentiel avec la moyenne européenne de 50\%. L'Espagne, cependant, bénéficie d'un niveau plus élevé de protection (dépenses par tête) que le Portugal.

\section{II- L'élargissement de 2004 : un défi pour le Portugal et l’Espagne.}

\section{1) Une concurrence accrue.}

Lors du Conseil européen qui se tint à Copenhague le 13 décembre 2002, l'UE ouvrit ses portes à l'Est et conclut des années de difficiles négociations avec dix pays candidats : l'Estonie, la Lettonie, la Lituanie, la Pologne, la République tchèque, la Slovaquie, la Hongrie, la Slovénie, Malte et Chypre. Ce sommet représentait le dernier acte de la réunification entre l'Ouest et l'Est à l'issue de la Guerre froide. Désormais, l'UE s'étendait du Portugal jusqu'aux frontières de la Russie, passant de 15 à 25 Etats en 2004, et atteignait une population de 451 millions d'habitants, un PIB de 8.800 milliards d'euros, et un PIB par habitant de 21.410 euros $^{25}$. Cet élargissement était le plus important en taille depuis la création de la $\mathrm{CEE}^{26}$, et, à l'inverse des précédents, comprenait d'anciens pays communistes où la société civile, les institutions indépendantes, une presse libre avaient été singulièrement absentes dès la fin de la Seconde Guerre mondiale ${ }^{27}$. Depuis la disparition de l'Union soviétique en 1991, ces pays ont construit de nouvelles institutions sociales, politiques et économiques. Le processus a été engagé, comme dans les cas espagnol et portugais, dans le but de rejoindre l'UE.

Or, l'élargissement a un grand impact et soulève toute une série de problèmes pour les pays ibériques. Certes, le Portugal et l'Espagne ont pu négocier des mécanismes compensatoires afin d'atténuer les conséquences négatives de traités d'adhésion qui leur sont défavorables, car il ne faut pas oublier que les deux pays ont bénéficié grandement des fonds européens : en 2001, l'Espagne, par exemple, percevait 63\% du budget consacré par l'UE aux fonds structurels (27,8 milliards d'euros). Mais parce que les pays ibériques sont les principaux

\footnotetext{
${ }^{25}$ Pierre Verluise, Géopolitique de l'Europe. L'Union européenne élargie a-t-elle les moyens de la puissance, Paris, Ellipses, 2005.

${ }^{26}$ Jean-François Drevet, L'élargissement de l'Union européenne. Jusqu'où ?, Paris, L'Harmattan, 2002.

${ }^{27}$ Jean-François Soulet, Histoire de l'Europe de l'Est de la Seconde Guerre mondiale à nos jours, Paris, Armand Colin, 2006, pp.218-241.
} 
bénéficiaires des fonds de redistribution de l'UE, l'entrée des pays d'Europe centrale et orientale (certains, comme la Pologne, étant largement agricoles) ne pouvait qu'entraîner une réduction des ressources attribuées aux pays ibériques, et en faire des contributeurs nets au budget européen. Ces fonds européens resteront les mêmes, au moins jusqu'en 2006, mais les gouvernements portugais et espagnol (ainsi que les acteurs économiques des deux pays) devraient être amenés à réévaluer les stratégies et les politiques courantes. Qui plus est, les pays les plus riches de l'UE ont déclaré leur intention de réduire les plafonds budgétaires actuels autour de $1 \%$ du PIB de l'UE, soit 815 milliards d'euros pour la période 2007-2013 28 . Dans le même temps, étant donné que les nouveaux Etats membres sont significativement plus pauvres, l'élargissement réduira le PIB moyen par tête de 10 à 20\%. Ainsi, le solde par habitant en Espagne et, à un moindre niveau, au Portugal, se rapprocheront de la moyenne de l'UE (c'est le fameux « effet statistique »), ce qui signifie que beaucoup de régions ibériques ne seront plus éligibles à l'aide européenne, les fonds partant vers les nouveaux Etats membres. En effet, avec les règles actuelles, seules les régions ayant un revenu par tête moyen de moins de $75 \%$ de la moyenne de l'UE (ce qui inclut pratiquement toutes les régions des nouveaux Etats membres) peuvent recevoir les fonds structurels en tant que régions de l'objectif 1. Or, avec l'élargissement, les disparités régionales doublent presque : 48 régions des Quinze (18\% de la population totale soit 68 millions d'habitants) avaient un revenu par habitant inférieur à $75 \%$ de la moyenne communautaire; dans une Union à 25, ces régions sont au nombre de 67 (25\% de la population soit 116 millions de personnes), les deux tiers d'entre elles se situant dans les nouveaux États membres. Seules 30 régions des États membres actuels (12\% de la population soit 47 millions) resteront ainsi sous le seuil des $75 \%$ et pourraient donc encore être considérées comme des « régions défavorisées », parmi lesquelles les Asturies, Murcie ainsi que Ceuta et Melilla pour l'Espagne, Madère pour le Portugal. Avec l'entrée de la Bulgarie et de la Roumanie en 2007 (les deux pays les plus pauvres de l'UE, avec un revenu par tête moyen de 27 et $26 \%$ de l'UE respectivement), on peut imaginer que le résultat sera encore plus faible.

Qui plus est, seuls les pays avec un revenu moyen de moins de $90 \%$ de la moyenne européenne ont accès aux fonds de cohésion, ce qui inclut tous les nouveaux pays (ainsi, le revenu moyen en Pologne représente $40 \%$ de la moyenne européenne, celui de la Lituanie 36\%, celui de Chypre 78\%). Une manne importante échappe ainsi aux pays ibériques (les fonds régionaux représentent à l'heure actuelle 34,5\% du budget de l'UE et la PAC 45\%). Comme résultat de l'élargissement, des groupes de pays apparaissent, en fonction d'un éventail de critères économiques de plus en plus large : un premier groupe, incluant les pays les plus pauvres, comprend huit des dix nouveaux membres (tous exceptés Chypre et la Slovénie), avec un revenu par tête moyen qui correspond à $42 \%$ de celui de l'UE, avec $21 \%$ de sa population; un second groupe est constitué de cinq pays (Chypre, Grèce, Portugal, Slovénie et Espagne), avec un revenu par tête moyen représentant $90 \%$ de l'UE et $13 \%$ de sa population; le dernier groupe comprend les pays les plus riches de l'UE, avec $66 \%$ de la population et un revenu par tête moyen de $115 \%$ par rapport à la moyenne européenne.

\section{2) Les risques de la marginalisation.}

Les pays de l'UE et la Commission discutent de mécanismes pouvant permettre une renégociation des différents fonds européens pour éviter leur disparition comme résultat de l'«effet statistique ». Les pays concernés proposent ainsi d'élever au-dessus des $75 \%$ les plafonds afin d'avoir accès aux fonds, ou d'établir des critères différents pour les pays membres. Néanmoins, il y a de fortes chances que dans le futur proche, les pays ibériques (surtout l'Espagne) deviennent contributeurs nets au budget de l'UE. Ainsi, les implications politiques, électorales (vote au Conseil) et budgétaires de ces développements seront très fortes pour les deux pays ${ }^{29}$.

De plus, l'Espagne et le Portugal devront mener rapidement la réforme de leurs structures économiques afin d'augmenter la productivité de leurs forces de travail, qui reste en dessous de la moyenne de l'UE. Comme conséquence du processus d'élargissement, le Portugal et l'Espagne auront à faire face à une concurrence grandissante sur leurs principales exportations hors agriculture - telles que les vêtements, les textiles et le cuir -, étant donné le niveau bas des salaires dans les Etats d'Europe centrale et orientale. Ces pays attireront l'investissement étranger dans des secteurs où l'Espagne et le Portugal, entre autres, ont été traditionnellement avantagés. Il est même probable que des usines produisant actuellement dans la péninsule seront tentées de se délocaliser à l'Est, ce qui s'est déjà produit avec le constructeur automobile Volkswagen, qui a choisi de réorienter une partie de sa production de la gamme Polo du site de Pampelune vers la Slovaquie ${ }^{30}$. Dans ce contexte, il sera important pour les pays ibériques de promouvoir une industrialisation misant davantage sur la qualité et la technologie.

\footnotetext{
${ }^{28}$ Benjamin Masse-Stamberger, «Budget européen : chacun pour soi », L’'Express, n 2816,20 juin 2005 , pp.120-121.

${ }^{29}$ D'où l'attitude intransigeante, parfois à la limite de l'agressivité, des pouvoirs espagnols à l'égard du problème du vote au Conseil.

${ }^{30}$ «VW délocalise la production de sa Polo à l'Est », Challenges, 25 avril 2006.
} 
L'élargissement, paradoxalement, pourrait favoriser ce processus parce qu'il fournira également des opportunités réelles aux pays ibériques, ainsi qu'aux autres Etats membres d'ailleurs. En effet, les produits portugais et espagnols ont désormais accès à de nouveaux marchés. Cette nouvelle donne devrait également permettre de diversifier les investissements ibériques à l'étranger: les entreprises espagnoles (et, à un moindre degré, portugaises) ont beaucoup investi en Amérique latine au cours de la dernière décennie; les incertitudes politiques et économiques actuelles dans cette région (que l'on pense à l'Argentine ou, pour des raisons différentes, au Venezuela) devraient entrainer une réorientation de l'investissement des entreprises ibériques vers les pays d'Europe centrale et orientale, qui sont sous-représentés par rapport à d'autres sociétés de l'UE : ainsi, les investissements espagnols vers la république tchèque sont passés de 37 à 3671 millions d'euros entre 2004 et 2005, et de 276 à 2187 en direction de la Hongrie ${ }^{31}$.

Les agriculteurs ibériques devront faire face également à de sérieuses difficultés du fait que l'appartenance à l'UE donne accès à la PAC pour les dix nouveaux Etats membres (quoique progressivement), et que les prix agricoles dans les pays en question (surtout en Pologne) sont en général plus bas que ceux de la péninsule. Quand les barrières opposées aux produits des PECO tomberont, les prix du marché en Espagne et au Portugal devraient s'effondrer. Qui plus est, pour certains produits, l'appartenance à l'UE va sûrement être un encouragement à produire plus dans les nouveaux Etats. Enfin, dans la prochaine décennie, la politique agricole sera réformée et sûrement réduite. Les dirigeants européens se sont mis d'accord en octobre 2002 pour geler les dépenses de la PAC jusqu'en 2013 ( $+1 \%$ à partir de 2006, ce qui se situe bien en-dessous des chiffres de l'inflation) ${ }^{32}$, ce qui a été confirmé lors du Conseil européen de Luxembourg (26 juin 2003); de plus, afin de faciliter l'élargissement (et le trade round de l'OMC à Doha), la Commission européenne a proposé le 27 janvier 2003 un plan pour réformer l'agriculture de l'UE, plan qui inclut : l'élimination des subventions liées à la production; le lien des subventions avec le fonds de développement rural; la réduction des paiements aux exploitations grosses et moyennes afin d'assurer une meilleure redistribution; l'arrêt des paiements aux agriculteurs qui ne respectent pas les nouveau critères sur la sécurité alimentaire, l'environnement et le bien-être animal ; enfin la réduction des prix que l'UE garantit pour certains produits. L'Espagne et le Portugal (mais aussi la France, l'Irlande, l'Italie, la Grèce et l'Autriche) sont réticents, car la réduction des fonds de la PAC entraînera le cofinancement des politiques agricoles, et de ceci les gouvernements portugais et espagnols ne veulent pas en entendre parler.

L'élargissement de l'UE va amener une réorientation de son centre de gravité vers le nord, et le «bloc méditerranéen » (incluant l'Italie et la Grèce), aux intérêts proches, aura ainsi moins de poids. Cette réorientation a des implications économiques et politiques sur les pays ibériques, en réduisant le pouvoir de veto du Portugal et de l'Espagne, tout en modifiant le cadre politique de l'UE. Dans une Europe à 25 membres, les pouvoirs institutionnels et l'influence de l'Espagne et du Portugal sont dilués, les votes de ces deux pays étant moins décisifs dans les institutions telles que la Commission, le Conseil, et le Parlement européen. Au total, le changement des priorités budgétaires et les moyens limités signifieront que certaines des politiques que l'Espagne et le Portugal ont défendues dans l'UE, comme le soutien à l'Amérique latine et l'Afrique du Nord (Maghreb), attireront moins l'attention et les ressources.

Ainsi, du point de vue de la convergence économique, étant donné le revenu actuel et les différentiels de productivité avec les pays les plus riches, les Etats ibériques sont obligés de poursuivre leur rattrapage à marche forcée. Pour cela, il est nécessaire que leurs économies prospèrent plus vite que celles des pays riches, ce qui demandera une libéralisation accélérée, ainsi qu'une concurrence grandissante au sein de leurs services, en même temps qu'une meilleure utilisation de leurs ressources productives. De plus, la convergence exige des réformes institutionnelles dans les politiques de Recherche et Développement, d'éducation, d'amélioration des infrastructures civiles, ainsi que plus d'innovation et d'investissement dans les technologies de l'information et des systèmes de formation plus performants. Une politique de convergence réussie nécessite un débat autour du rôle de l'investissement public et des programmes sociaux dans les deux pays, c'est-à-dire non seulement des stratégies et des politiques efficaces, mais également un engagement fort de la part des Espagnols et des Portugais à soutenir les réformes nécessaires.

III- Limites de la comparaison et leçons à méditer.

1) Un contexte difficile.

Des facteurs politiques ont été au cœur de la décision des Portugais et des Espagnols de rejoindre la CEE. Les deux pays voulaient renforcer leurs nouveaux régimes démocratiques et en finir avec l'isolement qui avait été le leur pendant la période autoritaire. Le Portugal et l'Espagne (ainsi que la Grèce) ont démontré combien étaient

\footnotetext{
${ }^{31}$ Source : registre des investissements extérieurs, Chambre franco-espagnole de commerce et d'industrie (http://www.lachambre.es).

${ }^{32}$ OCDE, Analyse de la réforme de la PAC de 2003, Paris, 2004.
} 
importantes les exigences démocratiques mises à leur candidature et combien ils ont fait d'efforts pour les remplir.

Les différences entre la situation des Etats ibériques en 1986 et celle d'aujourd'hui sont en effet flagrantes : les deux pays représentent de nos jours deux économies libérales avancées, des Etats forts et modernes, des sociétés civiles bien développées, avec syndicats et groupes d'intérêt, et profitent d'un haut niveau d'intégration entre deux économies liées par un faisceau d'intérêts communs. Quand on compare cette situation avec celle des pays de l'Est et que l'on confronte les défis que l'Espagne et le Portugal eurent à affronter dans les années 80 avec ceux qui attendent les nouveaux pays membres, ces derniers paraissent bien plus importants. En Europe centrale et orientale, les économies de marché sont en construction, et les démocraties restent fragiles ; aucun des pays candidats n'a d'expérience antérieure de la démocratie (exceptée la République tchèque); le Marché unique et l'UEM augmentent singulièrement les exigences portées pour toute nouvelle candidature; le climat politique et économique actuel, lui-même marqué par le 11 septembre et le fantasme de la mondialisation, rend l'élargissement encore plus difficile. Ainsi, intégrer la CE était plus simple en 1986 qu'aujourd'hui : depuis l'élargissement aux pays ibériques, le nombre de membres de l'UE a augmenté de 15, et les traités de Nice et d'Amsterdam (avec l'incorporation de nouveaux champs politiques tels que le pilier «justice et les affaires intérieures ») ont été ratifiés. Par conséquent, la complexité de l'acquis communautaire s'est accrue et les difficultés que les nouveaux membres ont à affronter sont nombreuses et variées. De plus, la culture différente et les expériences historiques diverses des nouveaux entrants vont compliquer le phénomène de convergence vers une identité européenne commune.

Comme dans le cas ibérique, les nouveaux membres ont transformé leurs systèmes politiques et économiques et adapté leurs règlements à l'acquis communautaire. Les coûts de cette transformation sont cependant bien plus élevés que dans le cas ibérique : le chômage a augmenté de $40 \%$ dans certaines régions, et les taxes jusqu'à $10 \%$, résultat de l'introduction de la TVA. Les transformations économiques et institutionnelles ont révélé les faiblesses des gouvernements et, dans de nombreux cas, cela a conduit au retour des anciens communistes au pouvoir, qui ont ralenti le train de réformes ${ }^{33}$. Qui plus est, les environnements économique et politique sont très différents et bien plus complexes. Le contexte international de l'adhésion fut un facteur important pour le succès de l'intégration ibérique ${ }^{34}$ : non seulement les économies de marché ibériques étaient en place (après une parenthèse révolutionnaire pour le Portugal), mais l'élargissement aux deux pays coïncidait avec la fin de la récession économique, la baisse des prix du pétrole, le début d'une période d'expansion au sein des économies européennes, et une nouvelle période de détente entre l'URSS et les Etats-Unis. L'économie actuelle (récession mondiale) et les incertitudes politiques (guerre contre le terrorisme, conflit du Moyen Orient, Irak, Corée du Nord), combinées aux difficultés économiques rencontrées par l'Allemagne et d'autres pays membres, rendent l'intégration bien plus difficile. En outre, étant donné l'envergure de l'élargissement et les disparités ainsi que les distances géographiques entre les nouveaux entrants, l'harmonisation risque de prendre du temps, tout en remettant au goût du jour le thème du « noyau dur », interprété par certains comme une forme de séparatisme occidental.

Pour les nouveaux membres, la modernisation économique sera le résultat d'un défi culturel, pas simplement la conséquence d'un processus d'ajustement comme dans les pays ibériques. Ainsi, les bénéfices économiques seront plus bas et mettront plus de temps à se matérialiser, alors que le nationalisme croissant de ces nouveaux entrants ralentira les effets culturels de l'intégration. Les nouveaux Etats membres auront à faire face à quatre grands problèmes internes ${ }^{35}:$ d'abord, la capacité à réformer leurs structures administratives et institutionnelles suivant les normes communautaires ; ensuite, la volonté de transformer leurs économies en économies de marché efficaces ; troisièmement, la capacité à réduire le chômage tout en luttant contre les déséquilibres économiques structurels ; enfin, les défis politiques qui consistent à réussir la transition vers l'adhésion malgré une opposition croissante aussi bien à l'intérieur qu'à l'extérieur. Les dix nouveaux entrants devront prendre en charge les réformes de leurs industries nationalisées, lutter contre la corruption, renforcer leurs systèmes judiciaires et leurs capacités à mettre en application les lois UE, éliminer certaines taxes litigieuses, en finir avec les avantages accordés au secteur public, réformer les finances publiques, privatiser des compagnies publiques démodées et immenses (ainsi, en Pologne, on compte 2000 entreprises d'Etat générant un quart de l'emploi et du PIB), renforcer la régulation financière, développer la transparence. Enfin, la PAC n'apportera pas que des subventions mais également plus de régulation et de concurrence.

Parallèlement, les niveaux de financement des politiques européennes (cohésion et fonds structurels, PAC) seront également plus limités pour les nouveaux Etats membres. Les traités d'adhésion, à la suite du sommet de Copenhague de 2002, promettent 40,8 milliards d'euros d'aide pour les dix entre 2004 et 2006 (auxquels il faut

\footnotetext{
${ }^{33}$ Guy Hermet et Lilly Marcou, Des partis comme les autres? Les anciens communistes en Europe de l'Est, Bruxelles, Complexe, 1998.

${ }^{34}$ A. Tovias, art. cit., p.175-176.

35 David R. Cameron, «The Challenges of EU Accession for post-communist Europe », Yale EU Studies,
} Lecture Series, 12 novembre 2002. 
ajouter un milliard d'aide régionale dans le cas polonais), ce qui représente seulement $0,15 \%$ du PIB de l'UE, ou autrement 22 euros par personne et par an pour les Quinze. Le transfert de fonds paraitra encore plus faible après la prise en compte des contributions des nouveaux Etats membres aux fonds de l'UE (estimées à 12 milliards d'euros, soit $4 \%$ du budget de l'UE et $0,05 \%$ de son PIB). Etant donné que le revenu par tête moyen des nouveaux Etats membres correspond à $44 \%$ de la moyenne européenne et leur PIB 5\% de celui de l'UE, ces fonds ne seront pas suffisants pour les aider à combler le fossé rapidement : avec ce niveau de soutien, cela devrait prendre beaucoup de temps pour le faire. Autrement dit, on ne pourra plus jamais atteindre les niveaux de l'aide européenne dans les cas grec, espagnol et portugais, niveaux qui sont pour beaucoup dans les progrès rapides de ces trois pays : la Grèce a ainsi reçu plus de 35 milliards d'euros de l'UE depuis son adhésion.

Le fait que les agriculteurs des nouveaux Etats doivent attendre 2013 pour recevoir le même soutien que ceux des autres membres (ils ont commencé à $25 \%$ des niveaux européens actuels en 2004) aggrave les difficultés. Cette situation est critique pour les pays dans lesquels l'agriculture représente encore une part importante dans l'économie nationale (deux Polonais sur dix sont des agriculteurs qui contribuent pour seulement 3,4\% du PIB national), dans le cadre d'un contexte économique difficile où les taux de chômage restent élevés $(19,6 \%$ en Pologne, 17,6 en Slovaquie, 12,4 en Lituanie en $\left.2003^{36}\right)$, de même que les déficits publics $(4,8 \%$ du PIB polonais, 4,5 du hongrois ${ }^{37}$ ).

Autre souci, le désenchantement croissant au sein des nouveaux Etats membres à propos de l'intégration européenne. Alors qu'il y a quelques années, l'adhésion était populaire dans l'opinion des pays candidats, la désillusion sur les conditions d'admission et le coût des réformes structurelles nécessaires à l'admission, ainsi que la peur d'être soumis aux capitaux et à l'influence étrangers ont généré un mécontentement, accroissant l'euroscepticisme et la montée de voix anti-UE. Des sujets sensibles de politique intérieure (chômage, impopularité des gouvernements), les subventions agricoles, Chypre, l'immigration, la polémique à propos des Sudètes, ou les possibles demandes d'anciens propriétaires expropriés par les nazis puis par les communistes dans certains pays (Silésie par exemple) ${ }^{38}$, tous ces sujets ont pris une nouvelle envergure avec le ralentissement économique et l'impact de certaines décisions liées à l'adhésion (par exemple, celles qui consistent à limiter les migrations pendant les sept premières années). Selon Eurobaromètre, le soutien à l'élargissement en a pâti avant même l'adhésion proprement dite ${ }^{39}$.

\section{2) Les principaux enseignements.}

Le processus d'élargissement ibérique éclaire rétroactivement le mécanisme actuel, non seulement en ce qui concerne les stratégies de négociation, mais aussi en termes de conséquences de l'adhésion pour les pays entrants. Quelles sont les leçons à en tirer ?

D'abord, aucune négociation (même avec l'UE) n'échappe à la maxime «diviser pour mieux régner », ce qui a parfois permis aux nouveaux Etats membres de tirer avantage des divisions entre les Etats déjà dans l'UE : la Pologne a par exemple suivi cette stratégie, notamment pour l'aide régionale. De plus, bien que les nouveaux membres (comme l'Espagne et le Portugal avant eux) partagent de nombreux problèmes (surtout les questions sociales, les fonds structurels, le concept de cohésion), chaque Etat membre devrait développer ses alliances en prenant soin d'éloigner le spectre d'un «bloc oriental» propre à bloquer tous les mécanismes. L'expérience ibérique montre que malgré des intérêts et des objectifs similaires, on ne peut pas parler d'une approche commune de l'UE entre l'Espagne et le Portugal : ils ont souvent collaboré, mais ils ont également travaillé séparément ou avec d'autres Etats membres.

Ensuite, l'une des leçons essentielles de l'élargissement ibérique concerne les termes de l'adhésion, qui ne sont jamais vraiment définitifs. Les renégociations après coup existent, et des mécanismes compensatoires sont souvent trouvés (le cas britannique le prouve également). Ainsi, quelles que furent les conditions négociées pour 2004-2006, l'objectif des nouveaux Etats membres porte bien au-delà de cette période, s'éloignant ainsi des perspectives budgétaires de l'UE, qui souhaite poursuivre la stabilisation et l'homogénéisation (comme le démontrent les réticences à propos du budget communautaire), alors que les nouveaux entrants veulent prospérer. A cet égard, l'expérience ibérique devrait amener les Etats de l'Est à chercher le meilleur moyen de maximiser les bénéfices de l'adhésion une fois à l'intérieur, notamment en se concentrant sur le thème de la cohésion économique et sociale.

\footnotetext{
${ }^{36}$ Eurostat, communiqué de presse 133/2004, « Le chômage dans l'UE25 ».

${ }^{37}$ Eurostat, communiqué de presse 39/2005, « données de déficit et de dette pour 2004 ».

${ }^{38}$ A signaler les travaux d'Anne Bazin, notamment «Effets et usages de l'intégration européenne sur la transformation des relations conflictuelles », Politique européenne, ${ }^{\circ}{ }^{\circ}$, hiver 2005-2006, pp.127-154, et « Les décrets Benes. De l'usage du passé dans le débat européen », Critique internationale, ${ }^{\circ} 21$, octobre 2003, pp.4251 .

${ }^{39}$ Sondage Eurobaromètre 2003-4, réf. IP/03/1722, 15 décembre 2003.
} 
En troisième lieu, le précédent luso-hispanique démontre les limites d'une pression trop forte et de la capacité de l'acquis communautaire à forcer le changement des mentalités et des pratiques. Ainsi, la Commission a insisté sur la nécessité de lutter contre la corruption et contre le crime économique, et de renforcer l'indépendance judiciaire dans les nouveaux pays membres ; cependant, certains analystes démontrent que cet effort devrait également concerner des pays déjà membres, parmi eux les pays méditerranéens, qui ont eu (et ont encore) des problèmes dans ces secteurs ${ }^{40}$. Etant donné les pesanteurs de l'histoire sur les pratiques administratives et économiques des pays ex-communistes, on ne peut pas tabler sur une transformation immédiate des mœurs suivant nos critères ${ }^{41}$. L'un des enseignements importants est que le succès économique entraîne le soutien populaire, mais ce dernier n'est pas inconditionnel et reste limité. Dans les cas espagnols et portugais, ce soutien est resté essentiellement opportuniste : le sentiment de «communauté européenne » est très faible, l'UE étant avant tout perçue comme une structure à vocation économique. L'UE ne doit donc se faire aucune illusion sur l'effet d'entraînement au niveau des esprits, sauf à faire montre d'une grande patience.

Par ailleurs, l'élargissement ibérique illustre le fait que les modèles migratoires peuvent être inversés. En effet, les deux pays durent attendre leur adhésion jusqu'en 1986, en partie à cause de peurs relatives à des mouvements de population qui ne se réalisèrent jamais. Au contraire, grâce à une situation économique améliorée, l'un des résultats principaux de l'adhésion à la CEE fut que, dix ans après, il y avait moins d'Espagnols et de Portugais vivant dans d'autres Etats de l'UE qu'avant l'élargissement; dans le même temps, des dizaines de milliers d'autres Européens venaient s'installer en Espagne. Aujourd'hui, malgré les peurs redoublées à propos de l'invasion de «plombiers polonais », la Commission européenne estime que seulement 70.000 à 150.000 travailleurs pourraient migrer de l'Est vers l'Ouest ${ }^{42}$. Les barrières de langues et de cultures devraient continuer à jouer leur rôle et à ralentir la mobilité. La croissance économique rapide de tous ces pays (particulièrement par rapport à des économies éprouvées comme en Allemagne) devrait avoir les mêmes conséquences que dans le cas ibérique : même si les migrations risquent d'entraîner des problèmes dans certaines régions frontalières (en particulier en Autriche et en Allemagne), il y aura plutôt peu d'émigration que beaucoup. Etant donné l'âge moyen à l'Ouest et les taux de fertilité, le mouvement pourrait même être encouragé.

Il est également important de noter que le soutien des fonds européens a largement influencé le succès des pays ibériques. Ces fonds ont favorisé des taux d'investissement public qui sont restés stables depuis le milieu des années 80. Le pourcentage d'investissement public de l'UE a atteint jusqu'à $42 \%$ au Portugal et $15 \%$ en Espagne ; de plus, la Commission européenne a estimé que l'impact des fonds structurels sur la croissance du PIB et l'emploi a été significatif (9,9 en Espagne et 3,1\% au Portugal). Ces fonds qui représentent le tiers du budget européen ont ainsi contribué à réduire les disparités régionales et à accompagner la convergence au sein de l'UE. Ils ont joué un rôle prééminent dans le développement de facteurs qui améliorent la compétitivité et déterminent la croissance des régions les moins développées des deux pays. Cela dit, le montant sera moins important pour les nouveaux Etats et plus difficile à capter, mais il peut tout de même entraîner une dynamique non négligeable.

Enfin, les difficultés que les économies ibériques ont connues au début des années 90 peuvent servir de leçon aux nouveaux membres : les «pilotes automatiques » ne fonctionnent pas. La crédibilité des autorités monétaires et économiques ne peut pas s'exprimer en rattachant ces dernières à des mécanismes institutionnels trop rigides, comme l'Espagne et, à un moindre degré, le Portugal ont essayé de faire, à la fin des années 80 et au début des années 90, en s'arrimant au SME. De plus, l'intégration des pays ibériques à l'UEM montre que la consolidation du processus d'intégration dépend d'une coordination adéquate des politiques économiques entre les membres avant l'adoption d'une monnaie. Au Portugal et en Espagne, l'intégration a exigé une série de mesures incluant une concurrence accrue, la privatisation des entreprises publiques, la restructuration industrielle et la dérégulation. Ces mesures se sont traduites en gains effectifs, qui ont été renforcés par un cadre macroéconomique plus stable. En même temps, une inflation plus basse et la consolidation fiscale ont amené des taux d'intérêt réel (et nominaux) plus bas, qui, à leur tour, ont soutenu une croissance plus forte. L'élargissement ibérique montre également qu'avant la priorité de l'intégration monétaire, les Etats nouveaux devront entreprendre un processus de modernisation. Une réforme financière n'induit pas nécessairement des changements institutionnels dans d'autres secteurs: l'échec du SME en 1992, causé en partie par plusieurs dévaluations successives, montre la limite des instruments financiers et monétaires à imposer des réformes institutionnelles. Celles-ci sont bien souvent le fruit d'une politique menée par des gouvernements qui acceptent de payer le prix de l'impopularité.

\footnotetext{
${ }^{40}$ C'est le constat que fait l'ONG Transparency International, notamment dans son dernier rapport Global Corruption Report 2006, consultable à l'adresse www.globalcorruptionreport.org/index.html.

41 Richard Backis, «L'héritage mental du soviétisme», in Pierre Verluise (dir.), Une nouvelle Europe. Comprendre une révolution géopolitique, Paris, Karthala, pp.151-162.

${ }^{42}$ Elarimpose, service européen de l'Assemblée des Chambres françaises de commerce et d'industrie, p.15, 2003, www.acfci.cci.fr/international/documents/elargissement2.pdf.
} 


\section{CONCLUSION :}

La dernière vague d'adhésions a beaucoup inquiété les opinions à l'Ouest, mais ces dernières ont largement surestimé certaines faiblesses des pays candidats, peut-être essentiellement par réflexe culturel : tout ce qui vient de l'Est continue à être sujet à caution, et il est demandé dans ce cas de figure un brevet d' " européanité » encore plus sévère, l'européisation étant assimilée à une forme d' «occidentalisation». Le passé, subrepticement, s'insinue dans cette réconciliation dite «historique », sans pour autant que chacun ait réellement médité les leçons de l'Histoire, les modes de légitimation définis par l'Ouest ne rencontrant pas toujours le besoin de restitution d'identité ressenti par certains pays candidats ${ }^{43}$. Chez les Occidentaux, la mauvaise conscience s'ajoute à une certaine fermeture d'esprit, et dans les pays de l'Est, l'attitude condescendante de l'Ouest est mal appréciée, d'autant plus que la rancune reste forte, entretenue par une forme de révisionnisme «victimisant ${ }^{44}$. Ajoutons un dernier mot : le contexte international n'est pas pour rien dans cette difficulté à «digérer» les pays de l'Est. L'ombre des Etats-Unis plane (personne n'a oublié que, dans les faits, c'est l'OTAN qui a été l'antichambre à l'appartenance à l'Union européenne), à une époque où certains pays, principalement occidentaux, rêvent de mettre en place une force politique et militaire européenne à part entière. Le 11 septembre et ses suites sont différemment interprétés de part et d'autre du Vieux continent, ce dont Washington profite amplement, démontrant une nouvelle fois combien il est difficile pour les Européens de trouver leur voie en toute autonomie.

Espagnols et Portugais n'ont pas eu à connaître la même situation, mais les ressemblances sont frappantes : l'état désastreux de l'économie, le passé autoritaire, les préjugés historiques, tout a déjà été entendu autour de 1986, lorsqu'il a été question d'accepter dans la CEE une partie, certes plus limitée, d'Europe. Toutefois, les différences restent importantes, la principale étant la reconnaissance à ces deux pays, sans débat particulier, d'une identité européenne non sujette à caution, que l'on n'a pas toujours volontiers accordée aux dix Etats candidats en 2004 (sans parler de la Roumanie et de la Bulgarie ni, bien entendu, de la Turquie). Ainsi, la situation économique périlleuse, qui semble résumer la difficulté des pays de l'Est à entrer dans les standards européens, n'est pas la seule explication à leurs problèmes d'intégration. Les cas espagnols et portugais démontrent que ces difficultés peuvent être surmontées, quitte à passer par certaines phases douloureuses.

Le problème, déjà en filigrane dans les cas ibériques, est ici beaucoup plus profond, et renvoie qui plus est, à des débats qui n'ont pas été tranchés parce qu'ils n'ont pas été abordés, étant encore tabous : la question de l'élargissement pose par contrecoup le problème des limites de l'Europe, et devra mécaniquement être éludé, à force de se poser. Ce problème n'est pas facile à régler, dans la mesure où il n'est pas que géographique, mais essentiellement mental, dans un continent mal limité par la nature, et qui a pourtant, plus qu'un autre, conscience de ce qui l'unit. Cette unité n'est donc pas un fait, mais doit être prouvée, et, plus encore, acceptée par tous les Européens : c'est là toute la difficulté qui se pose aux pays de l'Est, dont le chemin vers l'Europe est, contrairement aux apparences, loin d'être terminé ; c'est là également toute la complexité de leur quête, que rappellent les difficultés déjà éprouvées par les pays ibériques il y a un quart de siècle : l'élargissement n'est pas qu'un acte économique, mais bien politique, voire, plus encore, un acte de foi.

Bertrand VAYSSIÈRE, maître de conférences à l'université de Toulouse II- Le Mirail.

\footnotetext{
${ }^{43}$ Sur cette question, Gilles Pécout (dir.), Penser les frontières de l'Europe du XIXe au XXIe siècle, Paris, PUF, 2004.

${ }^{44}$ Georges Mink et Laure Neumayer (dir.), L’Europe et ses passés douloureux, Paris, La Découverte, 2007.
} 